\title{
A note on the welfare of the maximin rational expectations
}

\section{Zhiwei Liu}

Received: 15 November 2013 / Accepted: 5 May 2014 / Published online: 23 May 2014

(C) SAET 2014

\begin{abstract}
We provide a counterexample to the ex-ante efficiency of the maximin rational expectations equilibrium. In particular, we show that a maximin rational expectations equilibrium allocation may not be ex-ante maximin efficient, and therefore it may not be in the maximin core.
\end{abstract}

Keywords Maximin rational expectations equilibrium · Ex-ante efficiency

JEL Classification D51 $\cdot$ D61 $\cdot$ D80

\section{Introduction}

The interest of the maximin preferences of Gilboa-Schmeidler (1989), comes in that they take care of the cases where agents have difficulties to form a single prior. As a consequence, the Ellsberg paradox is resolved (de Castro and Yannelis 2013). Also, under the maximin preferences, the conflict between efficiency and incentive compatibility ceases to exist (de Castro and Yannelis 2010). That is, under the maximin preferences all (first-best) efficient allocations are incentive compatible. Furthermore, de Castro and Yannelis (2010) show that only maximin preferences have this property in general.

I am indebted to Nicholas C. Yannelis for his insightful comments and encouragements. I am also thankful to the anonymous referee for helpful comments and suggestions.

Z. Liu $(\bowtie)$

Department of Economics, The University of Illinois, Urbana-Champaign, USA

e-mail: liu108@illinois.edu 
The introduction of the maximin preferences into the general equilibrium modeling $^{1}$, enables us to have a rational expectations equilibrium notion that performs better than its Bayesian counterpart. Specifically, de Castro et al. (2013) show that the rational expectations equilibrium under maximin preferences (MREE) exists universally. The standard Bayesian rational expectations (REE) notion, however, exists generically [see Radner (1979) and Allen (1981)]. Indeed, in the Kreps (1977) example, a REE does not exist. However, de Castro et al. (2013) show that in the same example a maximin rational expectations equilibrium (MREE) exists, it is unique and interim efficient. Furthermore, Liu (2014) shows that this MREE allocation is maximin Nash incentive compatible and implementable.

In this note, we study the ex-ante welfare of the MREE notion ${ }^{2}$. We show that the MREE, which is interim efficient under the maximin preferences (de Castro et al. 2013), may not be ex-ante efficient. In particular, we show that there can be ex-ante welfare improvement switching from a MREE allocation to an alternative feasible, maximin Nash incentive compatible and implementable allocation. As a consequence, a MREE allocation may not be in the maximin core. That is, when deciding on an ex-ante insurance contract, a MREE allocation may not be a good candidate.

The note is organized as follows. In Sects. 2 and 3, we define an asymmetric information economy and the ex-ante efficiency notion. In Sect. 4, we present the counterexample to the ex-ante efficiency of the MREE notion. Finally, we conclude in Sect. 5.

\section{Asymmetric information economy}

Let $\Omega$ denote a finite set of states of nature, $\omega \in \Omega$ a state of nature, $\mathbb{R}_{+}^{\ell}$ the $\ell$ good commodity space, and $I$ the set of $N$ agents, i.e., $I=\{1, \ldots, N\}$. An asymmetric information economy $\mathcal{E}$ is a set $\mathcal{E}=\left\{\Omega ;\left(\mathcal{F}_{i}, \mu_{i}, e_{i}, u_{i}\right): i \in I\right\}$. Each element of the economy is common knowledge, unless specified otherwise. For each $i \in I, \mathcal{F}_{i}$ is a partition of $\Omega$. Let $E_{i}^{\mathcal{F}_{i}} \in \mathcal{F}_{i}$ denote an event, and $\omega \in E_{i}^{\mathcal{F}_{i}}$ a state in the event. Then, in the interim, if state $\omega$ occurs, agent $i$ observes the event $E_{i}^{\mathcal{F}_{i}}$, which is his private information. $\mu_{i}$ denotes agent $i$ 's prior. It is defined on events that are no finer than the ones in his information partition, that is, $\mu_{i}: \sigma\left(\mathcal{F}_{i}\right) \rightarrow[0,1]$, where $\sigma\left(\mathcal{F}_{i}\right)$ denotes the algebra generated by the partition $\mathcal{F}_{i}$. Note, if $E_{i}^{\mathcal{F}_{i}}=\left\{\omega, \omega^{\prime}\right\}$, then the probability of the event $E_{i}^{\mathcal{F}_{i}}$ is well defined, but not the probability of the event $\{\omega\}$ or $\left\{\omega^{\prime}\right\}$. The incompleteness of his prior is common knowledge $e^{3}$, but the prior is his private information. $e_{i}: \Omega \rightarrow \mathbb{R}_{+}^{\ell}$ is agent $i$ 's random initial endowment. Finally,

\footnotetext{
${ }^{1}$ For other applications of introducing the maximin preferences into the general equilibrium modeling, see Condie and Ganguli (2011), Correia-da-Silva and Herves-Beloso (2009), Dana (2004), de Castro and Chateauneuf (2011), and de Castro et al. (2011), just to name a few.

2 Glycopantis and Yannelis (2005) did the welfare analysis for the REE notion. They show that a REE may not be (pooled/full information) interim or ex-ante efficient.

${ }^{3}$ Consequently, it is common knowledge that if agent $i$ observes a non-singleton event in the interim, he does not have a probability assessment over the states in the event.
} 
$u_{i}: \mathbb{R}_{+}^{\ell} \times \Omega \rightarrow \mathbb{R}$ is agent $i$ 's ex-post utility function, taking the form of $u_{i}\left(c_{i} ; \omega\right)$, where $c_{i}$ denotes agent $i$ 's consumption.

Let $x_{i}: \Omega \rightarrow \mathbb{R}_{+}^{\ell}$ denote agent $i$ 's allocation (or in short, $i$-allocation). Denote by $L$ the set of all possible allocations of agent $i$, and by $x=\left(x_{1}, \ldots, x_{N}\right)$ an allocation of the above economy $\mathcal{E}$. An allocation $x$ is said to be feasible, if for each $\omega \in \Omega$, $\sum_{i \in I} x_{i}(\omega)=\sum_{i \in I} e_{i}(\omega)$.

In the interim, a state of nature $\omega$ is realized, agent $i$ observes the event $E_{i}^{\mathcal{F}_{i}}(\omega)$. He may even be able to refine his information to a smaller set, $E_{i}^{\mathcal{G}_{i}}(\omega) \subseteq E_{i}^{\mathcal{F}_{i}}(\omega)$, with the help of some public information revealed in the interim. Clearly, if no public information is released in the interim, or if the public information reveals less than what agent $i$ knows, then $E_{i}^{\mathcal{G}_{i}}(\omega)=E_{i}^{\mathcal{F}_{i}}(\omega)$.

An agent, who observes a non-singleton event in the interim, cannot form a probability assessment over the states of the nature in the event. This is due to the incompleteness of the private prior.

Following de Castro and Yannelis (2010), agents have maximin preferences, as axiomatized by Gilboa and Schmeidler (1989). That is, in the event $E_{i}^{\mathcal{G}_{i}}$, agent $i$ prefers the $i$-allocation $f_{i}$ to the $i$-allocation $h_{i}$ under the maximin preferences if

$$
\min _{\omega^{\prime} \in E_{i}^{\mathcal{G}_{i}}} u_{i}\left(f_{i}\left(\omega^{\prime}\right) ; \omega^{\prime}\right) \geq \min _{\omega^{\prime} \in E_{i}^{\mathcal{G}_{i}}} u_{i}\left(h_{i}\left(\omega^{\prime}\right) ; \omega^{\prime}\right) .
$$

Furthermore, under the maximin preferences, we say agent $i$ prefers $f_{i}$ to $h_{i}$ ex-ante if

$$
\sum_{E_{i}^{\mathcal{F}_{i} \in \mathcal{F}_{i}}}\left(\min _{\omega \in E_{i}^{\mathcal{F}_{i}}} u_{i}\left(f_{i}(\omega) ; \omega\right)\right) \mu_{i}\left(E_{i}^{\mathcal{F}_{i}}\right) \geq \sum_{E_{i}^{\mathcal{F}_{i} \in \mathcal{F}_{i}}}\left(\min _{\omega \in E_{i}^{\mathcal{F}_{i}}} u_{i}\left(h_{i}(\omega) ; \omega\right)\right) \mu_{i}\left(E_{i}^{\mathcal{F}_{i}}\right) .
$$

Agent $i$ strictly prefers $f_{i}$ to $h_{i}$, if he prefers $f_{i}$ to $h_{i}$, but not the reverse.

\section{Ex-ante efficiency}

We define the notion of the maximin core (see Liu and Yannelis 2013).

Definition 1 A maximin core allocation is a feasible allocation $x=\left(x_{i}\right)_{i \in I}$ for which there is no coalition $C \subseteq I, C \neq \varnothing$, and an allocation of the coalition $\left(y_{i}\right)_{i \in C}$ satisfying for all $\omega \in \Omega, \sum_{i \in C} y_{i}(\omega)=\sum_{i \in C} e_{i}(\omega)$, for all $i \in C, y_{i}$ is preferred to $x_{i}$ ex-ante under the maximin preferences, and for at least one $i \in C, y_{i}$ is strictly preferred to $x_{i}$.

When the coalition is the grand coalition, i.e., $C=I$, we have the ex-ante maximin efficiency notion. That is,

Definition 2 A feasible allocation $x=\left(x_{i}\right)_{i \in I}$ is said to be ex-ante maximin efficient, if there does not exist another feasible allocation $y=\left(y_{i}\right)_{i \in I}$, such that for all $i \in I$, $y_{i}$ is preferred to $x_{i}$ ex-ante under the maximin preferences, and for at least one $i \in I$, $y_{i}$ is strictly preferred to $x_{i}$. 


\section{Maximin rational expectations equilibrium (MREE)}

We explore the ex-ante efficiency of the MREE notion. The MREE notion of de Castro et al. (2013) is an interim solution concept. According to it, each agent chooses his consumption to maximize his interim payoff, taking into account both his private information and the information generated by the equilibrium prices. Here, unlike the standard rational expectations equilibrium notion of Radner (1979) and Allen (1981), the agents face ambiguity and have ambiguity aversion in the form of the maximin preferences.

Before stating the formal definitions, it is useful to introduce some notations.

A price vector $p$ is a non-zero function from $\Omega$ to $\mathbb{R}_{+}^{\ell}$. In a rational expectations equilibrium, agent $i$ 's interim information results from integrating his private information, $E_{i}^{\mathcal{F}_{i}}(\omega)$, with the public information revealed through prices, $p(\omega)$, where $\omega$ denotes the realized state.

Now, we describe $\mathcal{G}_{i}$, the set of possible interim information for agent $i$. Let $\sigma(p)$ denote the smallest algebra for which $p$ is measurable, and $\sigma\left(\mathcal{G}_{i}\right):=\sigma\left(\mathcal{F}_{i}\right) \vee \sigma(p)$, the smallest algebra containing both $\sigma\left(\mathcal{F}_{i}\right)$ and $\sigma(p)$, in which $\mathcal{G}_{i}$ is the partition of $\Omega$ that generates $\sigma\left(\mathcal{G}_{i}\right)$. In the interim, agent $i$ observes an event $E_{i}^{\mathcal{G}_{i}}(\omega)$ in $\mathcal{G}_{i}$.

Finally, given a price vector $p$, let $B_{i}(p ; \omega)$ denote agent $i$ 's interim budget set when the realized state is $\omega$,

$$
B_{i}(p ; \omega):=\left\{y_{i}: \Omega \rightarrow \mathbb{R}_{+}^{\ell} \mid y_{i}\left(\omega^{\prime}\right) \cdot p\left(\omega^{\prime}\right) \leq e_{i}\left(\omega^{\prime}\right) \cdot p\left(\omega^{\prime}\right), \forall \omega^{\prime} \in E_{i}^{\mathcal{G}_{i}}(\omega)\right\}
$$

Definition 3 A price vector $p$ and an allocation $x$ constitute a MREE for the economy $\mathcal{E}$ if:

1. for each $i \in I$ and for each $\omega \in \Omega, x_{i}(\omega) \cdot p(\omega) \leq e_{i}(\omega) \cdot p(\omega)$;

2. for each $i \in I$ and for each $\omega \in \Omega$,

$$
\min _{\omega^{\prime} \in E_{i}^{\mathcal{G}_{i}}(\omega)} u_{i}\left(x_{i}\left(\omega^{\prime}\right) ; \omega^{\prime}\right) \geq \min _{\omega^{\prime} \in E_{i}^{\mathcal{G}_{i}}(\omega)} u_{i}\left(y_{i}\left(\omega^{\prime}\right) ; \omega^{\prime}\right), \text { for all } y_{i} \in B_{i}(p ; \omega)
$$

3. and for each $\omega \in \Omega$,

$$
\sum_{i \in I} x_{i}(\omega)=\sum_{i \in I} e_{i}(\omega)
$$

de Castro et al. (2013) show that each MREE allocation is interim maximin efficient. We show that, for a MREE, there is room for improvement ex-ante.

Proposition A MREE allocation may not be ex-ante maximin efficient.

The example below is a modified version of the example 5.1 of Hahn and Yannelis (2001).

Example There are two agents, $I=\{1,2\}$, two goods, and three states of nature $\Omega=$ $\{a, b, c\}$. The ex-post utility function of each agent $i \in I$ is $u_{i}\left(x_{i, 1}(\omega), x_{i, 2}(\omega) ; \omega\right)=$ 
$\sqrt{x_{i, 1}(\omega) \cdot x_{i, 2}(\omega)}, \omega \in \Omega$, where the second index refers to the good. The agents' random initial endowments, information partitions and private priors are:

$$
\begin{aligned}
& \left(e_{1}(a), e_{1}(b), e_{1}(c)\right)=((7,1),(7,1),(4,1)) ; \mathcal{F}_{1}=\{\{a, b\},\{c\}\} \\
& \left(e_{2}(a), e_{2}(b), e_{2}(c)\right)=((1,10),(1,7),(1,7)) ; \quad \mathcal{F}_{2}=\{\{a\},\{b, c\}\} \\
& \mu_{1}(\{a, b\})=\frac{2}{3} ; \quad \mu_{1}(\{c\})=\frac{1}{3} \\
& \mu_{2}(\{a\})=\frac{1}{3} ; \quad \mu_{2}(\{b, c\})=\frac{2}{3}
\end{aligned}
$$

This economy has a fully revealing MREE. The price $p$ and the allocation $x$ are:

In state $\mathrm{a},\left(p_{1}(a), p_{2}(a)\right)=\left(1, \frac{8}{11}\right) ; x_{1,1}(a)=\frac{85}{22}, x_{1,2}(a)=\frac{85}{16}, x_{2,1}(a)=\frac{91}{22}$, $x_{2,2}(a)=\frac{91}{16}$.

In state $\mathrm{b},\left(p_{1}(b), p_{2}(b)\right)=(1,1) ; x_{1,1}(b)=4, x_{1,2}(b)=4, x_{2,1}(b)=4$, $x_{2,2}(b)=4$.

In state $\mathrm{c},\left(p_{1}(c), p_{2}(c)\right)=\left(1, \frac{5}{8}\right) ; x_{1,1}(c)=\frac{37}{16}, x_{1,2}(c)=\frac{37}{10}, x_{2,1}(c)=\frac{43}{16}$, $x_{2,2}(c)=\frac{43}{10}$.

Now, we shift some good 1 and good 2 from agent 1 to agent 2 under the state $a$, and define a new allocation $y$. Like the allocation $x$, the new allocation $y$ is meaningful. That is, both $x$ and $y$ are maximin Nash incentive compatible and implementable in the sense of Liu (2014).

At state a, $y_{1,1}(a)=\frac{80}{22}, y_{1,2}(a)=\frac{80}{16}, y_{2,1}(a)=\frac{96}{22}, y_{2,2}(a)=\frac{96}{16}$.

At state b, $y_{1,1}(b)=4, y_{1,2}(b)=4, y_{2,1}(b)=4, y_{2,2}(b)=4$.

At state $\mathrm{c}, y_{1,1}(c)=\frac{37}{16}, y_{1,2}(c)=\frac{37}{10}, y_{2,1}(c)=\frac{43}{16}, y_{2,2}(c)=\frac{43}{10}$.

Clearly, the allocation $y$ is feasible. Comparing with $x$, the allocation $y$ gives agent 2 strictly higher ex-ante maximin utility, without hurting the agent 1 .

Indeed, agent 2's ex-ante maximin utility under the allocation $y$

$$
\frac{1}{3} \cdot \sqrt{\frac{96}{22} \cdot \frac{96}{16}}+\frac{2}{3} \cdot \min \left\{\sqrt{4 \cdot 4}, \quad \sqrt{\frac{43}{16} \cdot \frac{43}{10}}\right\}=3.97
$$

is strictly higher than his ex-ante maximin utility under the allocation $x$

$$
\frac{1}{3} \cdot \sqrt{\frac{91}{22} \cdot \frac{91}{16}}+\frac{2}{3} \cdot \min \left\{\sqrt{4 \cdot 4}, \quad \sqrt{\frac{43}{16} \cdot \frac{43}{10}}\right\}=3.88
$$

While for agent 1 , his ex-ante maximin utility under the allocations $y$ and $x$ are the same

$$
\frac{2}{3} \cdot \min \left\{\sqrt{\frac{80}{22} \cdot \frac{80}{16}}, \quad \sqrt{4 \cdot 4}\right\}+\frac{1}{3} \cdot \sqrt{\frac{37}{16} \cdot \frac{37}{10}}=
$$




$$
\frac{2}{3} \cdot \min \left\{\sqrt{\frac{85}{22} \cdot \frac{85}{16}}, \quad \sqrt{4 \cdot 4}\right\}+\frac{1}{3} \cdot \sqrt{\frac{37}{16} \cdot \frac{37}{10}} .
$$

Consequently, the MREE allocation $x$ fails to be ex-ante maximin efficient.

Corollary A MREE allocation is not necessarily in the maximin core.

By definition, each maximin core allocation is ex-ante maximin efficient. Since a MREE allocation may not be ex-ante maximin efficient, a fortiori it may not belong to the maximin core.

\section{Concluding remark}

We showed that a MREE allocation may fail to be ex-ante maximin efficient, and therefore may not be in the maximin core. That is, the MREE allocations may not be good candidates for ex-ante insurance contracts. It is possible to improve the ex-ante welfare by switching from a MREE allocation to an alternative feasible, maximin Nash incentive compatible and implementable allocation.

\section{References}

Allen, B.: Generic existence of completely revealing equilibria for economies with uncertainty when prices convey information. Econometrica 49(5), 1173-1199 (1981)

Condie, S., Ganguli, J.V.: Informational efficiency with ambiguous information. Econ. Theory 48(2), 229242 (2011)

Correia-da-Silva, J., Herves-Beloso, C.: Prudent expectations equilibrium in economies with uncertain delivery. Econ. Theory 39(1), 67-92 (2009)

Dana, R.A.: Ambiguity, uncertainty aversion and equilibrium welfare. Econ. Theory 23(3), 569-587 (2004)

de Castro, L.I., Chateauneuf, A.: Ambiguity aversion and trade. Econ. Theory 48(2), 243-273 (2011)

de Castro, L. I., Yannelis, N.C.: Ambiguity aversion solves the conflict between efficiency and incentive compatibility (working paper) (2010)

de Castro, L.I., Yannelis, N.C.: An interpretation of Ellsberg's paradox based on information and incompleteness. Econ. Theory Bull. 1, 139-144 (2013)

de Castro, L.I., Pesce, M., Yannelis, N.C.: Core and equilibria under ambiguity. Econo. Theory 48(2-3), 519-548 (2011)

de Castro, L.I., Pesce, M., Yannelis, N.C.: A new perspective on rational expectations (working paper) (2013)

Gilboa, I., Schmeidler, D.: Maxmin expected utility with non-unique prior. J. Math. Econ. 18(2), 141-153 (1989)

Glycopantis, D., Yannelis, N.C.: Equilibrium concepts in differential information economies. Stud. Econ. Theory 19, 1-53 (2005)

Hahn, G., Yannelis, N.C.: Coalitional Bayesian Nash implementation in differential information economies. Econ. Theory 18, 485-509 (2001)

Kreps, M.D.: A note on fulfilled expectations equilibria. J. Econ. Theory 14(1), 32-43 (1977)

Liu, Z.: The implementation of the maximin rational expectations equilibrium allocations (working paper) (2014)

Liu, Z., Yannelis, N.C.: Implementation under ambiguity: the maximin core (working paper) (2013)

Radner, R.: Rational expectations equilibrium: generic existence and the information revealed by prices. Econometrica 47(3), 655-678 (1979) 